\title{
Biospeologica Sovietica L.: Une nouvelle espèce cavernicole de Harpolithobius (Chilopoda, Lithobiidae) du Caucase, Harpolithobius birsteini n.sp.
}

\author{
par
}

\section{N.T. ZALESSKAJA*}

Le matériel qui fait l'objet de cette note a été recolté en mai 1970 et en avril 1971 dans les grottes du Caucase (entre Soukhoumi et Akhali Afoni) pendant l'expédition de l'Institut d'Energie de Moscou. Les collections les plus riches ont été récoltées dans la grotte Avidzba, qui est à $10 \mathrm{~km}$ au Nord du village Verkhni Echery sur le versant occidental de la chaĩne de montagne de Skalny. Parmi celles-ci figurent notamment trois exemplaires d'une nouvelle espèce du genre Harpolithobius. C'est la première indication de la découverte de Harpolithobius dans des grottes d'URSS.

La nouvelle espèce est dédiée à la mémoire de l'éminent zoologiste et spéléologiste soviétique Professeur J.A. Birstein.

\section{Harpolithobius birsteini n.sp.}

Matériel: holotype I mâle (26.IV.71), paratypes 2 femelles (I.V.70); Abkhazie, grotte Avidzba, leg.Zalesskaja. Holotype et paratypes ont été déposés dans la collection du Muséum de Zoologie de l'Université de Moscou.

Description. Le corps est long de $18-20 \mathrm{~mm}$, coloré en jaune sale. La tête et les premiers articles de l'antenne colorés en jaune paille.

La tête est un peu plus large que longue, les ocelles sont peu distincts.

Antennes très minces et longues $(14-15 \mathrm{~mm})$ de 74 articles poilus chez le mâle et de 79-85 chez les femelles. Le dernier article antennaire est plus long que les deux articles précédents.

Les ocelles au nombre de 2 de chaque côté. Organe de Tömösvary elliptique et deux fois plus grand qu'un ocelle (fig.1).

Le coxosterne forcipulaire est beaucoup plus large que long (fig.2). Il est armé de $2+2$ petites dents triangulaires, et possède un porodonte puissant (fig. 3 ). Le bord rostral du coxosterne forcipulaire et les télopodites sont poilus, les griffes sont minces et longues.

Les tergites sont glabres, brillants et peu poilus. Les angles du bord postérieur des tergites I-VIII sont arrondis, ceux des X et XII sont droits, ceux du XIV aigus et ceux des IX, XI et XIII ont des prolongements triangulaires et larges. Quant au bord postérieur des tergites il est ou droit (tergites II, VI et VII) ou arrondi (IV), ou peu

\footnotetext{
* Institut d'Evolution, Morphologie et Ecologie animale, Académie des Sciences d'URSS, Vavilov Str. 26, MOSCOU, W-133, USSR.
} 
Tableau 1. Spinulation des pattes chez Harpolithobius birsteini n. sp.

\begin{tabular}{|c|c|c|c|c|c|c|c|c|c|c|}
\hline \multirow{2}{*}{$\mathrm{Nr}$. } & \multicolumn{5}{|c|}{ Ventral } & \multicolumn{5}{|c|}{ Dorsal } \\
\hline & $\mathrm{Cx}$ & $\operatorname{Tr}$ & Pf & F & $\mathrm{T}$ & $\mathrm{Cx}$ & $\operatorname{Tr}$ & $\mathrm{Pf}$ & $\mathrm{F}$ & $\mathrm{T}$ \\
\hline pp. & & & & & & & & & & \\
\hline 1 & - & - & $\mathrm{m}$ & $\mathrm{m}$ & $\mathrm{m}$ & - & - & - & $\mathrm{a}$ & $\mathrm{a}$ \\
\hline 2 & - & - & $\mathrm{mp}$ & $\mathrm{m}$ & $\mathrm{m}$ & - & - & $\mathrm{mp}$ & a & a \\
\hline 3 & - & - & $\mathrm{mp}$ & am & $\mathrm{m}$ & - & - & $\mathrm{mp}$ & a p & $\mathrm{a}$ \\
\hline 4 & - & - & $\mathrm{mp}$ & am & $\mathrm{m}$ & - & - & $\mathrm{mp}$ & a p & $\mathrm{a}$ \\
\hline 5 & - & - & $m p$ & $\mathrm{am}$ & $\mathrm{am}$ & - & - & amp & a $p$ & a p \\
\hline 6 & - & - & $\mathrm{mp}$ & am & $\mathrm{am}$ & - & - & amp & a $p$ & a p \\
\hline 7 & - & - & $\mathrm{mp}$ & $\mathrm{am}$ & $\mathrm{am}$ & - & - & amp & a p & a $p$ \\
\hline 8 & - & - & $\mathrm{mp}$ & $a m p$ & am & - & - & $\mathrm{amp}$ & a $p$ & a p \\
\hline 9 & - & - & $\mathrm{mp}$ & amp & $\mathrm{am}$ & - & - & amp & a $p$ & a p \\
\hline 10 & - & - & $\mathrm{mp}$ & amp & $\mathrm{am}$ & - & - & amp & a p & a $p$ \\
\hline 11 & - & - & $\mathrm{mp}$ & $\mathrm{amp}$ & $\mathrm{am}$ & - & - & amp & a p & a $p$ \\
\hline 12 & - & - & amp & ainp & $\mathrm{am}$ & - & - & amp & a p & a $p$ \\
\hline 13 & - & $\mathrm{m}$ & amp & amp & $\mathrm{am}$ & - & - & amp & a p & a p \\
\hline 14 & - & $\mathrm{m}$ & amp & amp & $\mathrm{am}$ & - & - & amp & a p & a $p$ \\
\hline 15 & - & $\mathrm{m}$ & amp & am & $\mathrm{am}$ & - & - & $\mathrm{am}$ & - & - \\
\hline
\end{tabular}

concave (I, III, V, VIII, X, XII et XIV) ou profondément concave (XV) et formé alors des lobes arrondis et poilus (fig.4). Les prolongements du tergite XIII ont de petites découpures. Les tergiter III, V, VIII, X, XII et XV sont beaucoup plus longs que larges; le tergite $\mathrm{X}$ est le plus grand.

Toutes les pattes sont longues et minces; la longueur des quatorzième et quinzième paires de pattes est d'environ $14 \mathrm{~mm}$. Le tibia de la quatorzième paire, chez le mâle, a un sillon dorsal qui comporte un groupe de soies sur une petite enflure située au tiers supérieur de ce sillon (fig.5). La griffe de la quinzième paire de pattes est double (fig.6). La spinulation des pattes est indiquée dans le tableau 1. $5 \mathrm{DPf}$ et 6 DPf peuvent avoir la spinulation mp.

Les pores coxaux sont arrondis, en nombre de 6-7-7-7 ou 6-7-7-6.

Les gonopodes de la femelle armés de $2+2$ éperons aigus et élargis, avec griffe apicale tridentée. Sur la face interne du premier article gonopodial se trouvent $4+2$

Fig. 1. Ocelles et l'Oragane de Tömösvary (OT)

Fig. 2. Coxosterne forcipulaire

Fig. 3. Bord rostral du syncoxite forcipulaire

Fig. 4. Tergite XV

Fig. 5. Tibia I4 chez le mâle

Fig. 6. Griffe apicale de PI5

Fig. 7. I article gonopodial vu de sa face interne

Fig. 8. Appendix genitale de la femelle 

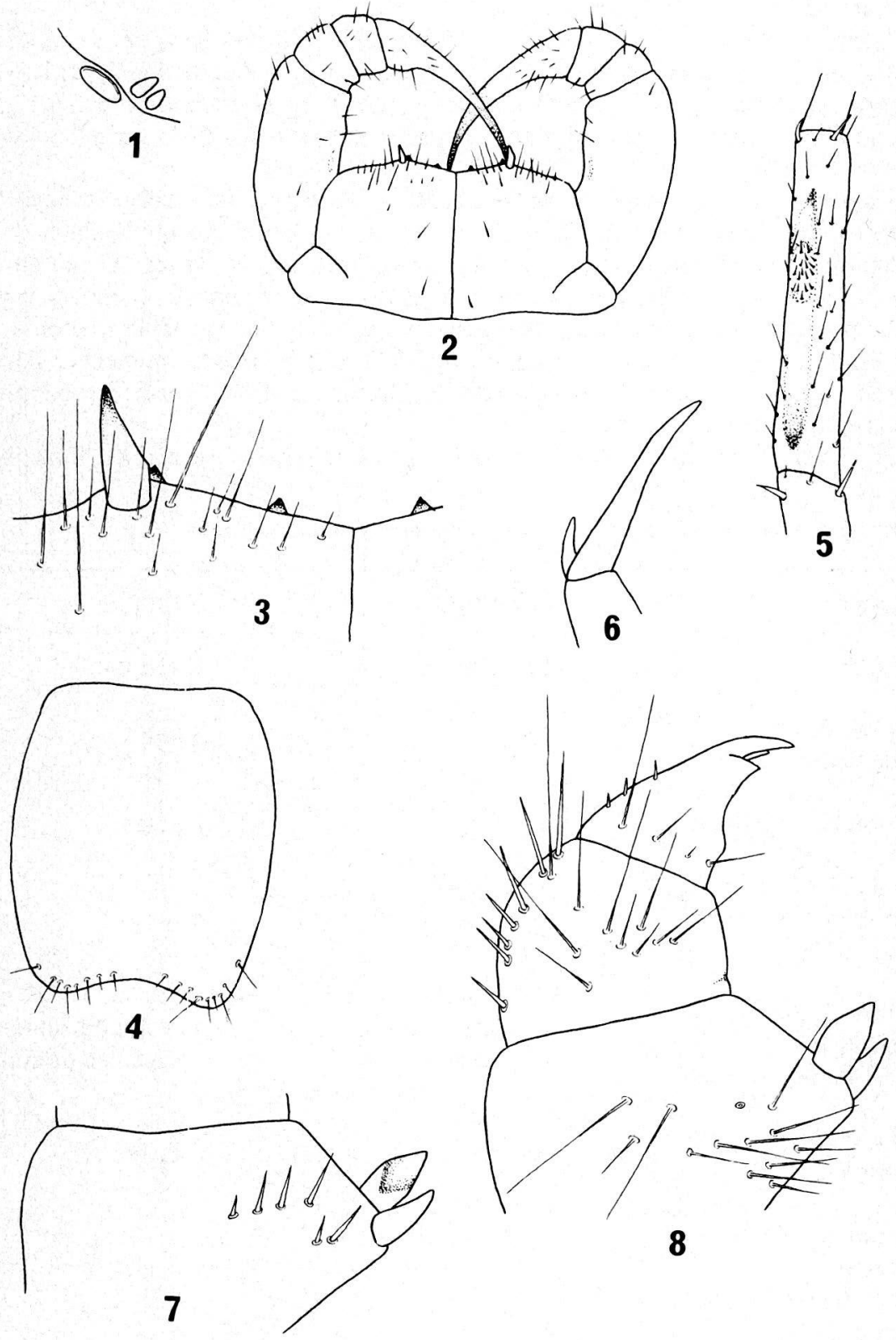
poils courts (fig.7); dorsalement, sur le deuxième article il y a 7 épines et sur le troisième article 3 petites épines (fig.8).

$H$. birsteini n.sp. est très proche de $H$. spinipes Folkm., 1958, qui a été décrit comme provenant de la région de Krasnaja Poljana; mais il s'en distingue par le petit nombre des ocelles, par les antennes qui sont plus longues et dont les articles sont plus nombreux, par quelques différentes portant sur les formations sexuelles secondaires etc.. (tabl.2).

Jusqu'ici parmi les espèces de genre Harpolithobius, une espèce aveugle $H$. oltenicus était considérée comme troglobie. Les particularités morphologiques de $H$. birsteini n.sp. (coloration jaune pâle du corps, antennes et pattes excessivement longues et minces, réduction du nombre des ocelles, organe de Tömösvary très grand) permettent de considérer aussi cette espèce comme un véritable troglobie.

L'étude des Lithobiides cavernicoles d'URSS est seulement commencée depuis peu, et aujourd'hui la faune des grottes du Caucase et de la Crimée est examinée plus attentivement que les autres.

En Caucase ont été trouvées 6 formes de Lithobiides cavernicoles: Lithobius

Tableau 2. Caractères différentiels de H.birsteini n.sp. et H.spinipes Folkm.

\begin{tabular}{|c|c|c|}
\hline Caractères & H.birsteini & H.spinipes \\
\hline Longueur & $18-20 \mathrm{~mm}$ & $11-18 \mathrm{~mm}$ \\
\hline $\begin{array}{l}\text { Nombre des artic- } \\
\text { les de l'antenne }\end{array}$ & $74-85$ & $49-59$ \\
\hline Nombre des ocelles & 2 & $15-20$ \\
\hline $\begin{array}{l}\text { Forme du bord } \\
\text { rostral du coxosterne } \\
\text { forcipulaire }\end{array}$ & $\begin{array}{l}\text { obtusangle } \\
\text { (concave) }\end{array}$ & droit \\
\hline $\begin{array}{l}\text { Enflure en } 3 \text {-eme } \\
\text { partie distale du } \\
\text { tibia } 14 \text { chez le mâle }\end{array}$ & $\begin{array}{l}\text { au bord du sillon } \\
\text { près de son fin }\end{array}$ & $\begin{array}{l}\text { au fond du sillon et } \\
\text { est reculée de son fin }\end{array}$ \\
\hline $\begin{array}{l}\text { Sillon du tibia } 15 \\
\text { chez le mâle }\end{array}$ & manque & présent \\
\hline $\begin{array}{l}\text { Nombre des épines } \\
\text { dorsales sur le } \\
\text { deuxième article go- } \\
\text { nopodial chez la } \\
\text { femelle }\end{array}$ & 7 & 4- 5 \\
\hline
\end{tabular}


vehemens Lign. (grotte Kazatchebrodskaja Pestera, région de Sotchi), L. erythrocephalus C. Koch (grotte Tsivtscala II, Géorgie), L. coloratus Ssel. (grotte Tsivtscala) L. viriatus Ssel. (grotte Azykhskaja Pestera en Nagornoo-Karabakhie), L. stuxbergi Ssel. (grottes Otapistavi, Vorontsovskaja Pestera et Chirokopokosskaja Pestera.) et Harpolithobius birsteini n.sp., décrite plus haut.

Dans les grottes de la Crimée sont connues à présent 3 espèces de Chilopodes seulement: L. skelicus Zal. (grotte Skelskaja Pestera), L. vehemens Lign. (grottes Krasnaja Pestera ou Kizil-Koba et Medovaja Pestera) et L. stuxbergi Ssel. (grottes Sundurlu, Khoii-Koba, Skelskaja Pestera et Krasnaja Pestera).

La majorité des Lithobiides, trouvée dans les grottes d'URSS, doit être considérée comme troglophile. Deux espèces seulement (L. skelicus et $H$. birsteini) sont des troglobies, Il est intéressant de noter que $H$. birsteini ainsi que $L$. skelicus (Zalesskaja, 1963) ont été trouvés dans une grotte deux fois au même endroit. Il n'est pas impossible de penser que les Lithobiides sont ainsi localisés parce qu'ils habitent dans les fissures des roches et en sortent pour gagner la grotte en des lieux précis et fixés. R. Jeannel (1926) a noté déjà cette particulariité pour quelques troglobies. Il a écrit que la prise répétée de troglobies au même endroit peut caractériser leur niche écologique à un certain degré. Nous pouvons confirmer cette opinion par nos deux exemples de Lithobiides du Caucase et de Crimée.

\section{RESUME}

Lors d'une récente exploration biologique des grottes du Caucase, une nouvelle espèce troglobie du genre Harpolithobius a été découverte dans la grotte Avidzba ( $10^{\star} 2$ 2 ) et sa description fait l'objet de cette note: Harpolithobius birsteini n.sp., c'est le deuxème Lithobiide troglobie connu des grottes de l'URSS, les autres espèces récoltées devant être seulement considérees comme troglophiles.

\section{SUMMARY}

A new troglobite species belonging to the genus Harpolithobius has been discovered during a recent biological exploration of Caucasian caves; $10^{\circ}$ and 29 were collected in Avidzba cave and are described here. Harpolithobius birsteini n.sp. is the second troglobiotic Lithobiid known from URSS caves. The other species collected must be considered as mere troglophilous.

\section{BIBLIOGRAPHIE}

FOLKMANOVA, B., 1958 - Nouvelles formes de Chilopoda de l'URSS, Comm. 2. Zool. J., XXXVII, 2: 183-192.

JEANNEL, R., 1926 - Faune cavernicole de la France. Encyclopédie Entomologique, VII, Paris.

ZALESSKAJA, N.T., 1963 - De Chilopoda cavernicole de la Crimée et du Caucase. Zool. J., XLII, 7: 1022-1030. 